This item was submitted to Loughborough's Research Repository by the author.

Items in Figshare are protected by copyright, with all rights reserved, unless otherwise indicated.

\title{
Superpixel based retinal area detection in SLO images
}

PLEASE CITE THE PUBLISHED VERSION

http://dx.doi.org/10.1007/978-3-319-11331-9_31

\section{PUBLISHER}

Springer International Publishing (๑ Springer International Publishing Switzerland)

\section{VERSION}

VoR (Version of Record)

\section{PUBLISHER STATEMENT}

This work is made available according to the conditions of the Creative Commons Attribution-NonCommercialNoDerivatives 4.0 International (CC BY-NC-ND 4.0) licence. Full details of this licence are available at: https://creativecommons.org/licenses/by-nc-nd/4.0/

\section{LICENCE}

CC BY-NC-ND 4.0

\section{REPOSITORY RECORD}

Haleem, Muhammad Salman, Liangxiu Han, Jano van Hemert, Baihua Li, and Alan Fleming. 2019.

"Superpixel Based Retinal Area Detection in SLO Images". figshare. https://hdl.handle.net/2134/20256. 


\title{
Superpixel based Retinal Area Detection in SLO Images
}

\author{
Muhammad Salman Haleem, Liangxiu Han, Jano van Hemert, Baihua li, and \\ Alan Fleming \\ School of Computing, Mathematics and Digital Technology, Manchester Metropolitan \\ University, Chester Street, Manchester, M1 5GD, UK \\ Optos plc, Queensferry House, Carnegie Business Campus, Enterprise Way, \\ Dunfermline, KY11 8GR, UK
}

\begin{abstract}
Distinguishing true retinal area from artefacts in SLO images is a challenging task, which is the first important step towards computeraided disease diagnosis. In this paper, we have developed a new method based on superpixel feature analysis and classification approaches for determination of retinal area scanned by Scanning Laser Ophthalmoscope(SLO). Our prototype has achieved the accuracy of $90 \%$ on healthy as well as diseased retinal images. To the best of our knowledge, this is the first work on retina area detection in SLO images.
\end{abstract}

Keywords: Scanning Laser Ophthalmoscope, fundus imaging, retinal image analysis, retinal artefacts extraction.

\section{Introduction}

Early detection and treatment of retinal eye diseases is critical to avoid preventable vision loss. Conventionally, retinal disease identification techniques are based on manual observations. Patients are imaged using a fundus camera or a Scanning Laser Ophthalmoscope (SLO). Optometrists and ophthalmologists often rely on image operations such as change of contrast and zooming to interpret these images and diagnose results based on their own experience and domain knowledge. Automated analysis of retinal images has the potential to reduce the time that the clinicians need to spend looking at images which can expect more patients to be screened and more consistent diagnoses can be given in a time efficient manner.

The 2-dimensional retinal scans obtained from imaging instruments (e.g. fundus camera, SLO) may contain structures other than retinal area; collectively regarded as artefacts. Exclusion of artefacts is important as a pre-processing step before detection of eye diseases. SLO has the widest FOV with over 200 degrees angle internal to the eye, which equates to over $82 \%$ of the retina visible unlike conventional fundus imaging, which can capture only 45 degrees. An example of result of both instruments is shown in Fig. 1. Due to the wide FOV of SLO images, extraneous objects such as the eyelashes, eyelids and dust on optical surfaces may appear bright and in focus. Therefore, automatic segmentation of 
these artefacts from SLO imaged retina is not a trivial task. The purpose of performing this study is to develop a method that can differentiate between retinal area and any artefacts present on ultra-wide field SLO images in order to segment out true retinal area.
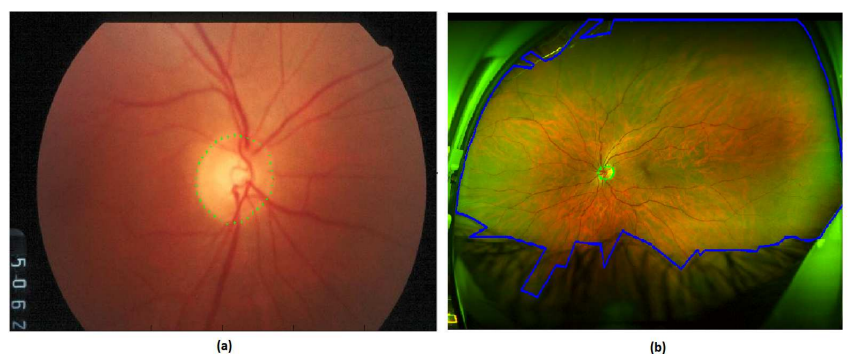

Fig. 1. An example of (a) a fundus image and (b) an SLO image annotated with true retinal area and Optic Nerve Head

To the best of our knowledge, there is no existing work to differentiate between retinal area and the artefacts. The main purpose of carrying out this study is to determine those features which can be used to distinguish between true retinal area and the artefacts. In this work, we have developed a new method to automatically extract out artefacts from SLO retinal scans which can be distinguished from retinal area using relevant image based superpixels. In order to make image analysis computationally efficient on high resolution SLO images, we have represented different small regions of SLO images as pixels. Our prototype has achieved the accuracy of $90 \%$ on healthy as well as diseased retinal images.

The rest of this paper is organised as follows: Section 2 introduces previous work for feature determination of fundus images, Section 3 discusses our proposed method, Section 4 provides the quantitative and visual results of our proposed method and Section 5 concludes the method with future work.

\section{Literature survey}

There are several image based features which have been represent different retinal structures in fundus images such as colour, illumination, intensity, skewness, texture, histogram, sharpness etc $[4,14,5]$. For reducing computational complexity, grid analysis containing small patches of the image has been proposed. [4] and the mean response of each feature aggregated over each patch was taken into account. The features of Region of Interest (ROI) of anatomical structures such as Optic Nerve Head (ONH) and Fovea have also been analyzed [10]. The features included structural similarity index, area and visual descriptor etc. Instead of grid analysis, the fundus retinal image have been divided into different 
irregular shaped subregions [13]. Some of the main features calculated for these subregions are Gaussian and its gradient, Difference of Gaussian etc.

Our current methodology is focused on using textural information to distinguish among retinal area and the artefacts. We divided each SLO image into small regions called superpixels. Superpixels represent the image in small meaningful regions and each region is equivalent to a pixel. The feature vector is calculated for each superpixel rather than pixel for high computational efficiency. For classification, we only used selected features so as to reduce the classification time. Since previously stated methods are applied on fundus images, our method is a first step for superpixel based image analysis in SLO images. The details of the methods are discussed in the following section.

\section{$3 \quad$ Methodology}

Our methodology is based on following steps:

- Image Preprocessing

- Superpixels Generation

- Feature Generation and Selection

- Classification

\subsection{Image Preprocessing}

Images were normalized by applying a $\operatorname{Gamma}(\gamma)$ adjustment to bring the mean image intensity to a target value. $\gamma$ was calculated using

$$
\gamma=\frac{\log _{10}\left(\mu_{\text {target }}\right)-\log _{10}(255)}{\log _{10}\left(\mu_{\text {orig }}\right)-\log _{10}(255)}
$$

where $\mu_{\text {orig }}$ is mean intensity of the original image and $\mu_{\text {target }}$ is mean intensity of the target image Finally, the Gamma adjustment of the image is given by Equation 2 .

$$
I_{\text {norm }}=\left(\frac{I}{255}\right)^{\gamma},
$$

\subsection{Superpixels Generation}

Previously, the superpixels have been generated for analyzing anatomical structures and retinal changes in fundus images [3,13]. For superpixel determination, Watershed approach is the quickest way for subregion determination. However, in order to avoid data redundancy, the superpixel generation method used in our methodology is Simple Linear Iterative Clustering (SLIC) [2], which was proved to be efficient in terms of computational time, region compactness and adherence. In SLIC, the image is sampled with initial clusters in a regular grid space. In the next step, each image pixel is associated with a nearest cluster centre within the search region, which is twice of the size of grid interval. The distance 
vector is calculated in terms of intensity values and pixel positions. After assigning each pixel to the nearest centre, an updating step adjusts the cluster centres to the mean of pixels in this group. The residual error is then calculated between new cluster centre and previous cluster centre. Such iterations continue until the error convergences.

\subsection{Feature Generation and Selection}

Table 1. Haralick Features

\begin{tabular}{|c|c|c|c|}
\hline Feature Name & Equation & Feature Name & Equation \\
\hline Autocorrelation & acorr $=\sum_{i} \sum_{j} i j p(i, j)$ & Mean Intensity & $I_{\mu}=\frac{\sum_{i} \sum_{j} I_{s}(i, j)}{N_{s}}$ \\
\hline Correlation & corr $=\frac{\sum_{i} \sum_{j}(i j) p(i, j)-\mu_{x} \mu_{y}}{\sigma_{x} \sigma_{y}}$ & Difference Entropy & $H_{\text {diff }}=-\sum_{i=0}^{N_{g}-1} p_{x-y} \log \left(p_{x-y}(i)\right)$ \\
\hline $\begin{array}{l}\text { Information Mea- } \\
\text { sures } 1\end{array}$ & $I M_{1}=\left(1-\exp \left[-2.0\left(H_{x y}-H\right)\right]\right)^{0.5}$ & Sum average & $\mu_{\text {sum }}=\sum_{i=2}^{2 N_{g}} i p_{x+y}(i)$ \\
\hline Sum Entropy & $H_{s u m}=-\sum_{i=2}^{2 N_{g}} p_{x+y} \log \left(p_{x+y}(i)\right)$ & $\begin{array}{l}\text { Sum of Squares: } \\
\text { Variance }\end{array}$ & $\sigma_{\text {sos }}=\sum_{i} \sum_{j}(i-\mu)^{2} p(i, j)$ \\
\hline Sum of Variance & $\sigma_{s u m}=\sum_{i=2}^{2 N_{g}}\left(i-H_{\text {sum }}\right) p_{x+y}(i)$ & Entropy & $H=-\sum_{i} \sum_{j} p(i, j) \log (p(i, j))$ \\
\hline $\begin{array}{l}p_{x+y}(k)=\sum_{i=1}^{N_{g}} \sum_{j=1}^{N_{g}} \\
1, \ldots, N_{g}, H_{x y}=-\end{array}$ & $\begin{array}{l}p(i, j), k=i+j-1=1,2,3, \ldots . \\
\sum_{i} \sum_{j} p_{x}(i) p_{y}(j) \log \left(p_{x}(i) p_{y}(j)\right)\end{array}$ & $\mathrm{V}_{g}$ and $p_{x-y}(k)=$ & $\sum_{i=1}^{N_{g}} \sum_{j=1}^{N_{g}} p(i, j), k=|i-j|+1=$ \\
\hline
\end{tabular}

After the generation of superpixels, the next step is to analyze their features. Due to textural difference between artefacts and retinal area observed in Fig. 1, one of the possible choices is the use of Haralick features [6] or Grey Level Co-occurrence Matrix (GLCM) analysis. GLCM analysis has been used for determining different regions in fundus retinal image [9]. It calculates second order statistics of an image using pixel adjacency. There are four angles for observing the pixel adjacency i.e. $\theta=0^{\circ}, 45^{\circ}, 90^{\circ}, 135^{\circ}$. The mean value in each direction was taken for each Haralick features. Also, Gaussian filter bank has been one of the most discriminative features for image based segmentation in fundus images [8, 13]. Gaussian filter bank includes Gaussian $\mathcal{N}(\sigma)$, its two first order derivatives i.e. $\mathcal{N}_{x}(\sigma)$ and $\mathcal{N}_{y}(\sigma)$ and three second order derivatives i.e. $\mathcal{N}_{x x}(\sigma), \mathcal{N}_{x y}(\sigma)$ and $\mathcal{N}_{y y}(\sigma)$ in horizontal(x) and vertical(y) directions. The filter bank is applied at scales $\sigma=1,2,4,8,16$.

We initially combined the Haralick features and Gaussian features and determined the classification power. The features are calculated for red and green channels (blue channel is zero in SLO images) and classification power was calculated using Area Under the Curve (AUC) [11]. AUC is taken using 5-fold cross validation on the training set. The classification power of complete feature set 
(40 Haralick and 60 Gaussian) is shown in Fig. 2(c). The feature vector of such a high dimension will affect the classification process in terms of computational efficiency therefore we decided to reduce its dimensionality.

In order to reduce the dimension as well as keeping eye on which features can be the part of feature set while having the classification power near to complete feature set, we used the following approach: From available set of features, the feature with highest AUC is selected. The next selected feature when combined with first selected feature, it will give highest AUC compared to other nonselected features. The process (shown in Fig. 2(d)) selected 10 features since AUC showed small improvement beyond it.
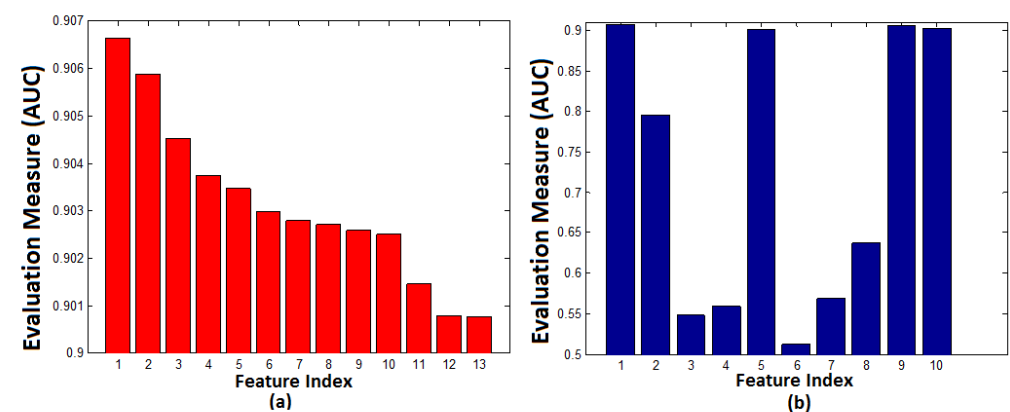

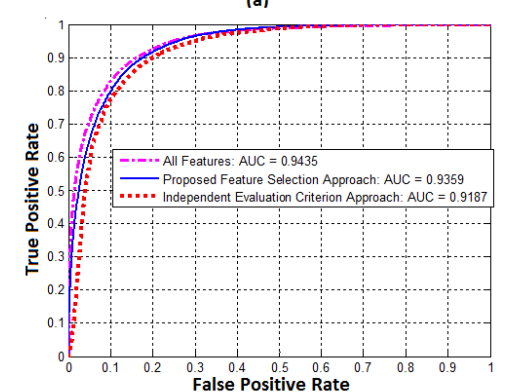

(c)

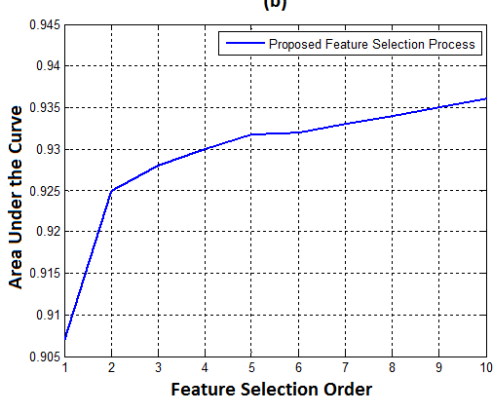

(d)

Fig. 2. (a),(b) Results of Independent Evaluation Criterion of IEC selected features and proposed feature set respectively. (c) Comparison of Classification power of all feature sets in terms of AUC and (d) Our feature selection process. The x-axis of both (b) and (d) is same and details mentioned in Table 2

The classification performance of proposed feature set was compared with feature set selected using Independent Evaluation Criterion (IEC) [7]. This approach ranks the features based on their Individual Classification Performance (ICP). We used AUC as evaluation criterion and selected the features with ICP greater than 0.9 . The ICP of 13 selected features under the criterion is presented 
in Fig. 2(a). We also evaluated ICP of the features selected by our proposed approach as shown in Fig. 2(b). The x-axis information of Fig. 2(a)(b) and (d) is presented in Table 2. The details of symbolic representation of Table 2 is presented in Table 1.

Compared to results in Fig. 2(a), ICP of most of the features in our proposed feature set were even below 0.8. Yet, the feature set was able to perform better with classification performance nearer to complete feature set (Fig. 2(c)). Also, unlike [8] and [13], Haralick features dominated in proposed feature set. The main drawback of Haralick features is its low computational efficiency in terms of pixel wise calculation but since we are using superpixels, the drawback has been addressed.

Table 2. Feature Selection Order of Fig. 2

\begin{tabular}{l|l}
\hline & Feature Symbols \\
\hline Fig. 2(a) & $\mathcal{N}_{R}(16), \mu_{\text {sumR }}, \mathcal{N}_{R}(8), \sigma_{\text {sumR }}, \mathcal{N}_{R}(4), \mathcal{N}_{R}(2), \mathcal{N}_{R}(1), \mathcal{N}_{y y R}(1), I_{\mu R}, \mathcal{N}_{x x R}(1)$, \\
& $\mathcal{N}_{y y R}(2), \operatorname{acorr}_{R}, \sigma_{\text {sos } R}$ \\
Fig. 2(b)(d) & $\mathcal{N}_{R}(16), \sigma_{\text {sosG }}, I M_{1 G}, \mathcal{N}_{y R}(16), \operatorname{acorr}_{R}, H_{\text {sum } R}, H_{\text {diff } R}, \operatorname{corr}_{R}, \mu_{\text {sum } R}, \mathcal{N}_{y y R}(1)$ \\
\hline
\end{tabular}

\section{Classifier Construction and Experimental Evaluation}

The main purpose of determining the feature set was determination of retinal boundary which can include large part of retinal area while keeping the artefacts out. Therefore, we constructed a classifier using Artificial Neural Networks [12] which takes training samples as inputs and determines the model that best fits to training samples using non-linear regression. The model was trained and tested for each of mentioned feature set so as to determine classification accuracy.

Table 3. Average Accuracies

\begin{tabular}{lll}
\hline Features Set & $D_{S}$ & $D_{P}$ \\
\hline Proposed Feature Selection & 90.81 & $92.00 \%$ \\
Independent Evaluation Criterion & 89.61 & $90.29 \%$ \\
\hline
\end{tabular}

The images for training and testing have been obtained from Optos [1] acquired using their ultra-wide field SLO. Each image has a dimension of $3072 \times$ 3900 and composed of only red and green channel. The dataset is composed of healthy and diseased retinal images; most of the diseased retinal images are from Diabetic Retinopathy patients. The system has been trained with 28 images and 
tested against 76 images. For training purpose, retinal mask covering the retinal area was applied after superpixel determination. The superpixels were assigned the class of retinal area or artefacts depending upon majority of pixels belonging to particular class.

The retinal area detection accuracy is determined using Dice Coefficient is the degree of overlap between the system output and the annotation mask obtained from the clinicians. The Dice Coefficient can be defined as in equation 3:

$$
D(A, B)=\frac{2|A \cap B|}{|A|+|B|}
$$

where $A$ and $B$ are the segmented images surrounded by model boundary and annotations from the ophthalmologists respectively, $|$.$| represents the area of$ the region, and $\cap$ denotes the intersection. Its value varies between 0 and 1 where a higher value indicates higher degree of overlap. The average superpixel classification accuracy $D_{S}$ and retinal area segmentation accuracy $D_{P}$ was calculated across both feature set and the results are shown in Table 3. As hypothesized on training set, the features selected by our proposed approach performed better on the test set as well. Some of the visual results on the test set are shown in Figure 3.
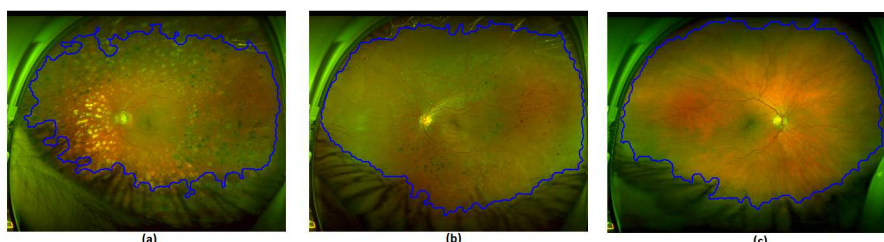

Fig. 3. Visual results of retina detector framework

\section{Conclusion}

Distinguishing true retinal area from artefacts in SLO images is a challenging task, which is also the first important step towards computer-aided disease diagnosis. In this work, we have proposed image based feature set for automatic detection of retinal area in SLO images. We have used superpixels to represent different irregular regions in a compact way and reduce the computing cost. A classifier has been built based on selected features to extract out true retinal area. The experimental evaluation result shows that image based features proposed by our methodology achieves an accuracy of $92 \%$ in segmentation of retinal area from SLO image. 
Since most artefacts detection methods have been applied previously to the fundus images, our method serves as a first step towards the processing of ultrawidefield SLO images. Moreover, a complete retinal scan is possible if the retina is imaged from different angles using an ultra-widefield SLO and then montaging the resulting image. Montaging can be possible only if the artefacts are removed before.

\section{References}

[1] www.optos.com

[2] Achanta, R., Shaji, A., Smith, K., Lucchi, A., Fua, P., Süsstrunk, S.: Slic superpixels compared to state-of-the-art superpixel methods. IEEE Transactions on Pattern Analysis and Machine Intelligence 34 (2011)

[3] Cheng, J., Liu, J., Xu, Y., Yin, F., Wong, D., Tan, N.M., Tao, D., Cheng, C.Y., Aung, T., Wong, T.Y.: Superpixel classification based optic disc and optic cup segmentation for glaucoma screening. IEEE Transactions on Medical Imaging 32, 1019-1032 (2013)

[4] Davis, H., Russell, S., Barriga, E., Abramoff, M., Soliz, P.: Vision-based, realtime retinal image quality assessment. In: 22nd IEEE International Symposium on Computer-Based Medical Systems. pp. 1-6 (2009)

[5] Haleem, M.S., Han, L., Hemert, J.v., Li, B.: Automatic extraction of retinal features from colour retinal images for glaucoma diagnosis: A review. Computerized Medical Imaging and Graphics 37, 581-596 (2013)

[6] Haralick, R.M., Shanmugam, K., Dinstein, I.: Textural features for image classification. IEEE Transactions on Systems, Man and Cybernetics 3 (1973)

[7] Liu, H., Motoda, H. (eds.): Feature Selection for Knowledge Discovery and Data Mining. Kluwer Academic Publishers Norwell, MA, USA (1998)

[8] Lupascu, C.A., Tegolo, D., Trucco, E.: Fabc: Retinal vessel segmentation using adaboost. IEEE Transactions on Information Technology in Biomedicine 14, 1267$1274(2010)$

[9] Muramatsu, C., Hatanaka, Y., Sawada, A., Yamamoto, T., Fujita, H.: Computerized detection of peripapillary chorioretinal atrophy by texture analysis. In: Conference Proceedings IEEE Engineering in Medicine and Biology Society. pp. $5947-5950$ (2011)

[10] Pires, R., Jelinek, H., Wainer, J., Rocha, A.: Retinal image quality analysis for automatic diabetic retinopathy detection. In: 25th SIBGRAPI Conference on Graphics, Patterns and Images (SIBGRAPI). pp. 229-236 (2012)

[11] Serrano, A.J., Soria, E., Martin, J.D., Magdalena, R., Gomez, J.: Feature selection using roc curves on classification problems. In: The International Joint Conference on Neural Networks (IJCNN). pp. 1-6 (2010)

[12] Smola, A., Vishwanathan, S.: Introduction to Machine Learning. Cambridge University Press (2008)

[13] Tang, L., Niemeijer, M., Reinhardt, J., Garvin, M.: Splat feature classification with application to retinal hemorrhage detection in fundus images. IEEE Transactions on Medical Imaging 32, 364-375 (2013)

[14] Yu, H., Agurto, C., Barriga, S., Nemeth, S.C., Soliz, P., Zamora, G.: Automated image quality evaluation of retinal fundus photographs in diabetic retinopathy screening. In: IEEE Southwest Symposium on Image Analysis and Interpretation (SSIAI). pp. 125-128 (2012) 